\title{
LA EVALUACIÓN POR COMPETENCIAS Y SUS IMPLICACIONES PEDAGÓGICAS 1991-2004'
}

\author{
MARTHA JEANETH CASTILLO BALLÉN*
}

\section{Resumen}

Este artículo pretende mostrar, a manera de síntesis, cuáles son las exigencias de la evaluación por competencias en el área de lenguaje, de acuerdo con las políticas estipuladas por el Instituto Colombiano para el fomento de la Educación Superior (ICFES); qué se ha logrado y qué falta por lograr en el ambiente educativo con el fin de alcanzar el ideal de una educación que pretende resignificar la interacción en el aula.

Palabras clave: evaluación, lenguaje, significación, comunicación, interacción, conocimiento y educación.

\footnotetext{
Abstract

This article pretends to show, in a synthetic way, which are the demands of the evaluation by competences in the area of language, per the policies that are provided by the Colombian Institute for The Promotion of Higher Education (Instituto Colombiano para el Fomento de la Educación Superior (ICFES); what has been achieved and what needs to be achieved in the educational ambient in order to reach the ideal of an education that pretends to gibe a new meaning to the interaction at the classroom.

Keywords: evaluation, language, meaning or signification, communication, interaction, knowledge and education.
}

\section{LA EVALUACIÓN POR COMPETENCIAS EN LENGUAJE} (SNE, 1997 Y 1999) ${ }^{2}$

En Colombia, desde comienzos de la década del noventa, la evaluación por competencias en lenguaje ha estado inscrita en el marco de una reflexión teórica sobre el lenguaje desde la cual se postula que el proceso de significación de lo humano es una condición indispensable para lograr la formación integral de los sujetos en las diferentes dimensiones de su desarrollo: social, cognitivo, cultural, estético y físico (Baena, 1992 y 1989) ${ }^{3}$.

Según el profesor Luis Ángel Baena, la significación es el proceso según el cual se transforma la experiencia humana en sentido; transformación que se expresa en términos de categorías concep-

\footnotetext{
${ }^{1}$ Estudio iniciado durante el primer semestre del 2003 por parte del Área de Lenguaje de la Subdirección Ácadémica del ICFES, cuyo propósito es analizar los presupuestos disciplinares y pedagógicos que subyacen a la evaluación por competencias en el área de lenguaje.

* Licenciada en lingüística y literatura de la Universidad Distrital de Colombia. Estudios de maestría en literatura, Instituto Caro Cuervo. Profesional especializada, Área de Lenguaje, Subdirección de Aseguramiento de la Calidad del ICFES. Correo electrónico: mcastillo@icfes.gov.co.

${ }^{2}$ Consultar estas obras para profundizar en las discusiones y desarrollos conceptuales en la evaluación por competencias que se han venido dando desde hace más de una década en el Grupo de Lenguaje de la Subdirección de Aseguramiento de la Calidad de la Educación del ICFES

${ }^{3}$ Orientación dada, en el ámbito educativo, por el profesor Luis Ángel Baena en la década de los ochenta, y cuyo pensamiento sirvió de base para la formulación del enfoque semántico comunicativo.

ARTículo RECIBIDo 30 DE MAYO DE 2004. ACEPTADo 13 DE JUNIO DE 2004
} 
tuales, pragmáticas y culturales. De acuerdo con este enfoque, la apropiación de la lengua se percibe como el resultado de la integración progresiva del niño a una comunidad verbal. Es en la interacción con el mundo como toma conciencia de sí mismo, del otro y del mundo natural y social que lo circunda; se integra a la vida como participante en la negociación de sentidos, desde las más tempranas etapas de su desarrollo cognitivo.

En este contexto, más que tomarse como un sistema de reglas o un instrumento de la comunicación, el lenguaje se concibe como un hecho social que constituye al hombre como sujeto cultural y discursivo (Berger y Luckmann, 1968; Silvestri y Blank, 1993; Bruner, 1988). Un sujeto que se construye en su experiencia individual y colectiva con el mundo a través del lenguaje, cuya actividad se expresa en textos y discursos.

Esta orientación supedita el análisis del sistema de la lengua al proceso de la significación, a la construcción y búsqueda del sentido a través del uso, a los elementos que intervienen en el proceso de interacción y la acción discursiva. En consecuencia, en el plano educativo exige una pedagogía en la que el desarrollo del lenguaje y la construcción

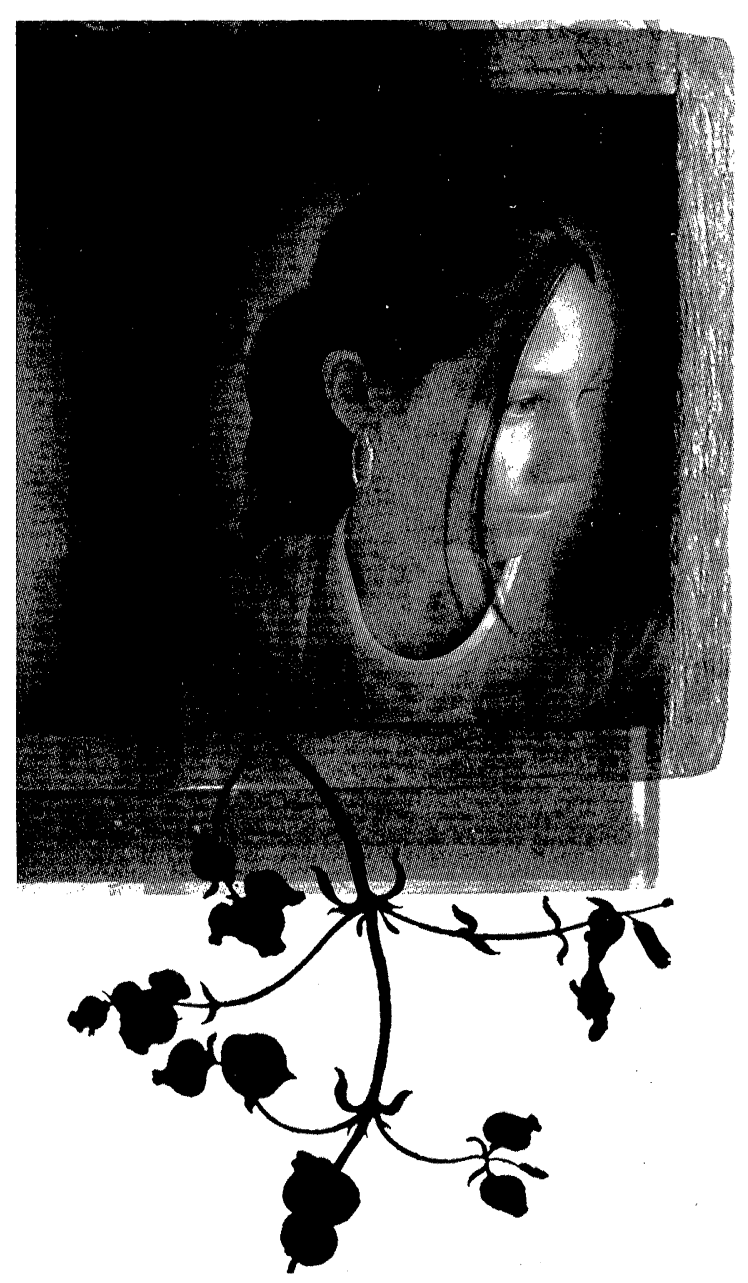

de saberes aparezcan en una misma dimensión, ya que es con y a través del lenguaje como el estudiante construye y desarrolla conocimiento, como significa sus experiencias y le da sentido a las experiencias de otros. Desde esta línea teórica se le apuesta a una noción de conocimiento en la que el lenguaje es el elemento esencial puesto que estructura y comunica conocimiento.

Asumir esta responsabilidad supone tomar conciencia sobre el papel que juega el lenguaje en los procesos de socialización en la escuela y por fuera de ella, y la manera como los individuos interpretan y significan el mundo. Atendiendo a estas exigencias, y siendo conscientes de la necesidad de apoyar desde la evaluación la construcción de estos espacios pedagógicos, las pruebas de Estado aplicadas por el ICFES pretenden evaluar los estados en la competencia comunicativa de los estudiantes a través de la lectura y la producción de textos.

Para tales propósitos, el ICFES entiende por competencia comunicativa la capacidad que tiene un estudiante para comprender, interpretar, organizar y producir actos de significación a través de distintos sistemas de signos lingüísticos y no lingüísticos; un "saber-saber-hacer" con el lenguaje, en contextos específicos ${ }^{4}$.

La decisión de rastrear el estado de la competencia comunicativa de los estudiantes a través de la lectura y la escritura respondió a la convicción de que sus logros no podían determinarse únicamente con base en el dominio de un cuerpo de contenidos sobre el funcionamiento gramatical de la lengua, aprendidos por lo general de manera memorística y descontextualizada ${ }^{5}$. Por el contrario, el estudiante evidencia el estado de su competencia comunicativa cuando consigue utilizar el lenguaje (y para algunos, su lenguaje) en interacciones exitosas e interpreta o produce textos atendiendo no sólo a las reglas del sistema gramatical, sino a

\footnotetext{
${ }^{4} \mathrm{Si}$ el conocimiento se percibe como un proceso en continua transformación y estructuración en y por el lenguaje y no como una bolsa de contenidos, entonces la evaluación debe volver la mirada hacia el proceso del conocer. En este sentido, cuando decimos que la competencia es un "saber-saber-hacer" estamos sustentando la posibilidad de una acción, de un hacer inmerso en ese proceso de desarrollo gradual, que se cumple en la construcción y apropiación de herramientas que posibiliten la transformación de la experiencia humana en sentido.

${ }^{5}$ Esto no significa desconocer la importancia del conocimiento teórico sobre el lenguaje y la literatura, lo que se intenta cuestionar es el aprendizaje de reglas y conceptos, sin que en ese proceso de aprendizaje exista una conciencia funcional del lenguaje.
} 
las condiciones pragmáticas de la enunciación en contextos particulares.

Los anteriores planteamientos, propuestos por primera vez en el campo de la evaluación en 1991, no han sufrido mayores modificaciones. Sin embargo, su implementación en el nuevo Examen de Estado y las recientes aplicaciones de las Pruebas Saber han señalado la necesidad de hacer ajustes en el diseño de los instrumentos, introducir cambios en la interacción en el aula y reorientar la formación de docentes. En esa dirección, el marco teórico de evaluación se ha venido enriqueciendo con aportes de la sociolingüística, la psicolingüística, la texto-lingüística, la semiótica y las teorías contemporáneas de la recepción ${ }^{6}$.

En consecuencia, la evaluación como proceso orientado a la transformación de las prácticas educativas pretende desarrollar en estudiantes y profesores la voluntad de saber, el deseo de aprender a aprender para lograr la autonomía e incidir con sus acciones, razonadas y críticas, en la resolución de conflictos.

\subsection{El proceso de lectura y escritura en el marco dle la evaluación por competencias}

Frente a las teorías que conciben la interpretación como la identificación de la intención del autor en el texto o a las que la entienden como seguimiento de la intención del lector, la teoría semiótica de la recepción de Umberto Eco (1965, 1972, 1977, 1981, 1985 y 1988) afirma la necesidad de buscar en el texto lo que dice, con referencia a los sistemas de significación desde los que fue emitido y a su propia coherencia interna. Desde esta perspectiva, la libertad interpretativa del lector es estimulada y regulada por el texto. El destinatario de un texto llena los espacios vacíos que el texto contiene, realizando un recorrido por sus diferentes niveles con base en los conocimientos que el texto le exige y en los movimientos interpretativos que éste, además, motiva en él.

La reflexión que sustenta esta teoría tiene como fundamento la semiótica y específicamente el concepto de signo de Charles S. Peirce (1987), para quien hablar de signos es responder a la pregunta por el conocimiento. En efecto, a la pregunta ¿cómo conoce el hombre?, este autor responde: a través de signos. El signo en Peirce es el resultado de un proceso de interpretación; un signo nos ofrece una versión del mundo, que a su vez debe ser interpretada por otros signos, en un proceso de semiosis. Así, interpretar un signo es relacionarlo con otros que, según el contexto y el universo de discurso, sirven para aclarar o ampliar su significado y sentido. Aplicada a la comunicación y específicamente al proceso de lectura, esta concepción se resuelve en una teoría de la cooperación interpretativa que busca distinguir entre interpretación y uso del texto.

En este proceso de cooperación interpretati$\mathrm{va}^{7}$ entre los saberes del texto y los saberes del lector, el estudiante se vale, de manera progresiva y regulada por el texto, de conocimientos previos ${ }^{8}$, de representaciones sobre la manera como se perciben e interpretan experiencias, saberes que apuntan a las diferentes relaciones entre sujetos y eventos del mundo, saberes conceptuales sobre temas determinados, situaciones de enunciación particulares. En este intercambio de saberes, conocidos y por conocer, es como el lector va construyendo hipótesis de lectura acerca de lo que puede decir el texto.

Este proceso de interacción, en las Pruebas Saber y de Estado, actúa como un abanico de posibilidades interpretativas. En un primer momento, las hipótesis del lector son amplias y diversas, debido a que los conocimientos que se activan obedecen, de manera casi arbitraria, a la percepción que se hace el lector de los posibles contenidos textuales ${ }^{9}$. A medida que el lector avanza en su proceso de interpretación, este abanico se va estrechando, para dar paso al descarte o la constatación de ciertas hipótesis, o para considerar otras que hasta el momento no se habían vislumbrado. Posteriormente, el abanico se abre nuevamente para interactuar con otros textos que la lectura convoca

\footnotetext{
${ }^{6}$ En esta línea teórica han trabajado autores como M. M. Bajtín, Emile Benveniste, Gérard Genette, Teun A. van Dijk., A. J. Greimas, Oswald Ducrot, y Umberto Eco, entre otros.

${ }^{7}$ En términos de la semiótica discursiva, se diría que es una interacción entre los códigos desde los cuales lee el sujeto y los códigos desde los cuales el texto prevé sus lecturas, a la memoria de otros textos.

${ }^{8}$ Se hace referencia a la competencia enciclopédica del estudiante, es decir, al repertorio total de todos los saberes que el estudiante ha logrado aprehender en su interacción con el mundo.

${ }^{9}$ Es necesario aclarar que este aspecto nos remite a la capacidad del lector de elaborar conjeturas e hipótesis razonables sobre el contenido del texto a partir de sus saberes previos. Estos saberes pueden ser hipercodificados o hipocodificados, dependiendo de las exigencias del texto. Son hipercodificados cuando el texto remite a saberes altamente socializados e hipocodificados cuando el texto exige la interpretación de saberes que requieren de un metalenguaje que no es altamente socializado.
} 
como intertextos o voces que se articulan a lo dicho o sugerido. En este juego de conjeturas, de aciertos y desaciertos, de generalizaciones y abstracciones, de encuentros y desencuentros es como el lector construye el sentido del texto.

A medida que se avanza en el proceso de interpretación, el lector tiene una exigencia de selección de saberes que van desde los más cercanos e inmediatos a su mundo, hasta los conceptuales y específicos de un metalenguaje. Cada texto hace una exigencia de saberes pertinentes a su estructuración y significados posibles ${ }^{10}$.

En el proceso de evaluación, la cooperación interpretativa entre texto y lector se ve mediada por un grupo de preguntas que, a partir de la organización de cada texto, apuntan a marcar diferentes recorridos de significación del contenido textual, exigiendo del lector un trabajo en función de una hipótesis de lectura global que le permitirá responder a las preguntas: ¿Qué dice el texto? ¿Para qué lo dice? ¿Cómo lo dice? ¿Quién lo dice? Si el lector reconoce lo que dice el texto, podrá entrar a responder la segunda pregunta: ¿Qué pienso yo sobre lo que dice este texto en relación con otros textos?

Es un trabajo que exige pasar del trabajo textual (interpretación semántica) al trabajo intertextual (interpretación semántica crítica) para, posteriormente, dar paso al trabajo extratextual (interpretación crítica o propositiva) ${ }^{11}$. Entendemos por interpretación semántica el resultado del proceso por el cual el lector, ante la manifestación lineal del texto, la llena de significado; y por interpretación crítica, el proceso mediante el cual el lector intenta explicar por qué razones estructurales el texto puede producir esas u otras interpretaciones.

En términos de Genette (1989), la intertextualidad regula el proceso de interpretación entre la experiencia del lector y las exigencias del texto, en cuanto da cuenta de la presencia efectiva de un texto en otro. Es importante anotar que en este proceso el estudiante pone en juego sus conocimientos sobre el lenguaje, la literatura y otras disciplinas. En el diálogo con los textos, se vale de sus lecturas previas para avanzar, en una lectura relacional, a niveles de interpretación críticos.

En el ICFES se entiende por texto toda estructura significante de signos verbales o no verbales en la que sus elementos sintácticos, semánticos y pragmáticos conforman una red de significación en continua interacción, en función de un sentido global y de una estructura particular, que es la que diferencia un texto de otro. El texto y el discurso podrían ser considerados como dos elementos diferentes, que negocian y convergen en el mismo proceso de la significación. En efecto, para una semiótica cuyo objeto son las prácticas significantes, el discurso es el proceso de significación y a la vez el acto que envuelve el proceso de la enunciación. El texto, por su parte, es el que permite organizar y expresar la significación del discurso ${ }^{12}$.

Ahora bien, el proceso de interacción entre texto y lector se evidencia cuando el lector consigue producir interpretantes de ese texto. Se considera interpretante cualquier nuevo signo que, desde cierta perspectiva, interpreta o explicita, los contenidos del texto. Por ejemplo, sus ilustraciones, sus resúmenes, sus comentarios críticos, sus adaptaciones a otras sustancias de la expresión e, incluso, los efectos emotivos que pueda producir en su receptor. Es de anotar que, más allá de la infinidad de interpretantes que pueden darse de un texto, éste no admite cualquier interpretante y será fundamental distinguir cuándo el interpretante producido por el lector da cuenta del texto o cuándo lo tergiversa.

En el marco de este enfoque, la escritura es considerada como un proceso individual y a la vez colectivo, en el cual' se configuran mundos a través de la puesta en juego de saberes, experiencias y estrategias de significación. La escritura apunta a la negociación, consciente o inconsciente, entre texto y discurso, a un sentido global en el que se manifiestan, de manera explícita o implícita, una intención comunicativa y unas circunstancias enunciativas particulares ${ }^{13}$.

En este proceso, como bien lo dice Barthes $(1973)^{14}$ ocurre la elección del área social en el seno

\footnotetext{
${ }^{10}$ En esta medida se podría llegar a decir que la complejidad de cada texto estaría determinada por la calidad del proceso lector, es decir, por el carácter exitoso de la comunicación: exigencias del texto vs. saberes, capacidades y experiencias del lector.

${ }^{11}$ Categorías ampliamente discutidas por Umberto Eco (1995).

12 En este sentido, los conceptos de coherencia y cohesión estarían haciendo referencia al proceso de negociación entre texto y discurso, ya que la coherencia apunta a la orientación intencional del discurso, y la cohesión a la organización del texto para lograr la puesta en escena del discurso.

${ }^{13}$ Para un análisis más profundo sobre los aspectos sociales y pragmáticos que subyacen en el proceso de la escritura, ver Jurado y Bustamante (1997).

14 Sobre el proceso de escritura como proceso semiótico se puede consultar a Barthes (1973), o la obra de Ong (1987).
} 
de la cual el escritor decide situar la naturaleza de su lenguaje. En el ejercicio de la escritura se construye lo que aparece de manera natural y regulada en el proceso de interacción entre dos personas. ¿A quién le estoy escribiendo? ¿Para qué? y ¿cómo hacerlo?; son algunos de los interrogantes que se responden en el acto mismo de la producción y que permiten establecer una correlación entre el plano de la expresión y el contenido. Este proceso, al igual que la interpretación de un texto, reclama una argumentación que le imprima al texto cierta autonomía. En la acción de la escritura se estructura un mundo y se modelan los enunciadores y posibles enunciatarios.

Si en el proceso de comprensión el estudiante trabaja por develar las estrategias textuales y discursivas para poder dialogar con el texto, en el proceso de producción escrita debe construirlas para lograr que otros puedan interactuar con él. Esto exige un conocimiento básico de las estrategias proporcionadas por el lenguaje como instrumento de la significación ${ }^{15}$.

Producir un texto es construir sentido, configurar un escenario, sus actores y relaciones, sus posibles espectadores y críticos, a través de la acción discursiva. En este sentido, el trabajo sobre los procesos de coherencia y cohesión presupone un saber previo de estrategias de organización textual que se movilizan en el acto mismo de la escritura: construcciones sintácticas, semánticas y pragmáticas que se seleccionan y organizan de una manera particular, atendiendo al sujeto textual que se construye en la dinámica de la producción. En este sentido, la escritura, al igual que la lectura, es un proceso generador de conocimiento.

Atendiendo a los procesos de significación que se espera propiciar mediante una educación que centra el desarrollo del lenguaje en la lectura y construcción de textos, las preguntas abiertas o de producción escrita tienen como propósito, más que ver el problema de corrección gramatical, la capacidad para producir significados a través de diferentes tipos de textos. Es evidente que los dos problemas no pueden separarse tajantemente (no hay forma de producir significados sin acogerse a una gramática), pero sí pueden ser valorados de maneras diferentes: problemas de corrección gramatical, como la ortografía o la concordancia, pueden llegar a determinar la coherencia de un texto, pero no al mismo nivel del desarrollo de un tema.
Asumimos, entonces, que en la producción de los estudiantes es posible distinguir entre una estructura profunda o universo de sentido y una estructura superficial, en la que se mediatiza ese universo de sentido. Al evaluar el texto producido por el estudiante, se pretende caracterizar la coherencia y las estrategias utilizadas para hacerlo.

Planteada de esta manera, aunque la observación de preguntas abiertas puede parecer un procedimiento discutible, la posibilidad de determinar problemas puntuales con el objeto de evaluarlos en cada aplicación (correferencias, manejo de enlaces lógicos, usos funcionales de los signos de puntuación, conservación de tópicos temáticos, por ejemplo), sin pretender agotar en un solo intento lo que se presenta como una lista abierta, nos permite ir avanzando y profundizando en el tipo de competencias que debe afianzar la escuela para lograr que el proceso de escritura sea un proceso consciente hacia la construcción de saberes.

Como se verá más adelante, para abordar la calificación de estas preguntas se construyen unas rejillas que sirven de marco o parámetro de evaluación. En estas rejillas se consignan los aspectos relacionados con el proceso de producción de los niños y se definen posibles actuaciones. Luego, al analizar los resultados, se buscan esos recorridos $\mathrm{u}$ otros que no habían sido previstos y se definen tendencias dominantes.

\subsection{Lo deseado y lo esperado en el marco de uma formación por competencias en lengua materna}

En este marco conceptual ¿cuál es el nivel deseado en la formación en lenguaje en la educación básica y media y qué resultados arroja la evaluación al terminar estos ciclos?

Un estudiante que ha terminado su educación básica y media debe tener conciencia del uso del lenguaje en diferentes contextos, ser capaz de comprender, interpretar, analizar y producir tipos de textos según sus necesidades comunicativas y las exigencias del medio cultural, social y académico que lo rodea, y de adoptar comportamientos multipolares, analíticos e integrales en la generación y adquisición de conocimientos. Debe ser una perso-

\footnotetext{
${ }^{15}$ Sobre el tema de las estructuras textuales y discursivas, consultar a Dijk (1980). Sobre el proceso de escritura como proceso semiótico, ver Barthes, (1973) y Ong (1987).
} 
na que pueda responder no sólo a los retos que la sociedad le va a exigir, sino a su propia actitud hacia la vida y sus posibilidades de seguir aprendiendo.

Si éste es el nivel de competencia esperado, entonces una mirada sobre el proceso de lectura y escritura, tal como se evidencia en la evaluación, nos permitirá caracterizar estados o momentos que den cuenta, a manera de diagnóstico, de lo que se está logrando y de lo que faltaría por lograr en los proyectos educativos y de aula, con el fin de conseguir que la práctica pedagógica, en el área de lenguajé, se convierta en un hacer cada día más significativo.

A partir de las conclusiones parciales a las que se ha llegado en los análisis anteriores, en este último capítulo se explican los resultados de los estudiantes en las diferentes pruebas; así mismo, se plantean algunas sugerencias que apuntan al mejoramiento de la calidad de la educación en lenguaje.

\section{UNA MIRADA GLOBAL}

\section{A LA EDUCACIÓN BÁSICA}

$\mathrm{Al}$ analizar los resultados obtenidos en las últimas pruebas aplicadas a los estudiantes de educación básica y media, se puede decir que en el proceso escolar la lectura y escritura sigue siendo una actividad anexa al proceso de construcción de conocimientos: se le enseña al estudiante a leer y escribir, pero no a aprender leyendo y escribiendo. La

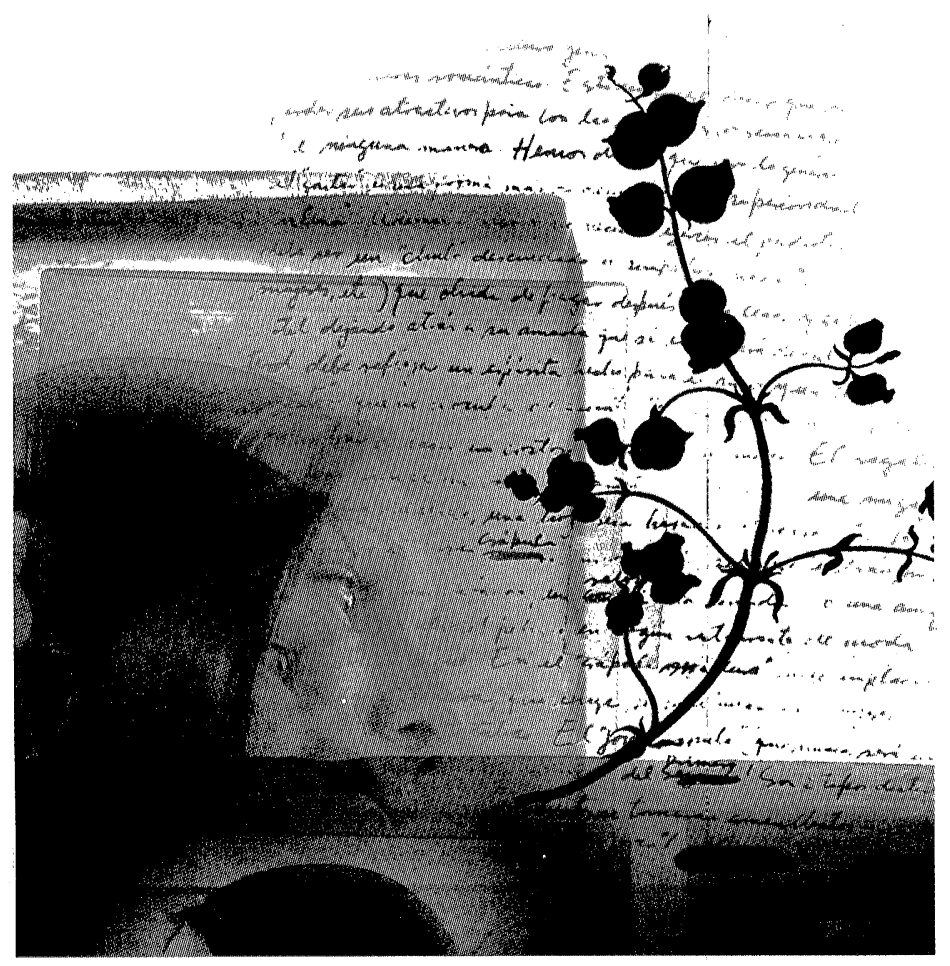

mayoría de los estudiantes de este nivel realizan procesos de semantización básicos, caracterizados por lecturas en las que prima una comprensión fragmentaria y localizada de la información contenida en los textos, lo que no les permite acceder a lecturas en las que se intente responder al por qué se dice lo que se dice y para qué se dice.

Cuando el estudiante logra responder a la pregunta sobre ¿qué dice el texto?, y luego genera relaciones con lo que conoce del tema o de su autor, está configurando un marco de comunicación que le permite relacionar un texto con otros, con lo cual está en capacidad de descubrir las voces y discursos que atraviesan el texto como espacio de la interacción. El movilizar saberes de un texto a otro ayuda a crear una actitud favorable hacia el conocimiento, ya que el estudiante tiene la posibilidad de proponer un nuevo texto sobre lo leído (es aquí donde adquiere sentido la toma de notas en clase o la elaboración de un trabajo escrito). Cuando las lecturas sólo se quedan en un resumen literal, se le está negando al estudiante la posibilidad de ser partícipe en la construcción del conocimiento.

Los análisis anteriores indican que se necesita superar la dificultad para acceder a niveles de lectura, especialmente, en los siguientes casos:

(i) Cuando se ponen en juego los componentes textuales y discursivos de un texto. (ii) Cuando se movilizan saberes del texto al lector y del lector al texto. (iii) Cuando se relacionan universos de sentido desde diferentes perspectivas.

La posibilidad de formar lectores críticos depende de la oportunidad que tenga el estudiante para superar la típica tarea de recuperar lo que dice el texto de manera literal o extraer las ideas centrales del mismo.

En este sentido, las acciones educativas deberían estar encaminadas a propiciar el paso de la lectura literal hacia la lectura inferencial, y de ésta hacia la lectura crítica-intertextual. Es de vital importancia flexibilizar el acceso a los textos, presentarle al estudiante diversidad de textos, cualificar los niveles de lectura en los maestros y en los padres de familia, como los principales actores en el proceso educativo de un niño.

Los análisis realizados sobre los desempeños de los estudiantes en las diferentes pruebas permiten decir que éstos realizan un proceso de co- 
operación interpretativo crítico, cuando los textos remiten a una información cercana a sus códigos y que son muy pocos los que realizan este mismo tipo de lectura, cuando los textos exigen, primero, una experiencia en el manejo de estructuras narrativas y argumentativas un poco más complejas $y$, segundo, movilizar saberes que requieren de un apoyo conceptual.

Si queremos lectores críticos, es necesario que en la escuela, y desde muy temprano, se le brinde al estudiante la posibilidad de leer diferentes tipos de textos, de complejidades diversas y que inciten a aprender leyendo. Los textos de información científica, periodísticos (sobre cultura, sociedad y economía), al igual que los ensayos sobre literatura o sobre otros temas, permiten que el lector haga un uso integral de sus conocimientos.

Los resultados sugieren la importancia de trabajar tanto en la básica como en la media para superar los niveles de lectura literal que no aportan herramientas en la construcción de conocimientos ni permiten que el estudiante sea un sujeto crítico y propositivo, teniendo presente que las dificultades en las pruebas se relacionan con:

- Inferencias enunciativas: relaciones entre qué se dice, quién lo dice y para qué se dice. Relación enunciador-enunciado-enunciatario.

- Inferencias léxicas: cadenas semánticas que permiten relacionar la información local en busca de un sentido global.

- Inferencias referenciales: reconocer y hacer un uso funcional de los elementos que permiten la progresión temática.

- Inferencias macroestructurales: selección, clasificación y jerarquización de ideas.

- Inferencias lógicas: relaciones lógicas entre las ideas de un texto. Un trabajo textual que involucra los elementos gramaticales en la coherencia y construcción de sentido.

- Inferencias argumentativas: reconocer y usar las relaciones que se generan, tipos de argumentos: inducción-deducción, razonamiento causal, razonamiento dialéctico.

- Relaciones enciclopédicas e intertextuales: movilizar información de su enciclopedia académica o cotidiana para enriquecer e interpretar otros textos.

- Lecturas relacionales: cómo un texto se menciona en otro texto y cómo se presenta ese diálogo de voces.

Los aprendizajes evaluados en lenguaje trascienden la mera presencia o ausencia de un deter- minado concepto o tema. Lo que la prueba evalúa es la manera como el estudiante hace uso de estos saberes para comprender e interpretar textos verbales y no verbales.

\section{POSIBLES EXPLICACIONES}

\section{A LOS PROBLEMAS ENCONTRADOS EN LAS PRUEBAS}

¿Qué hay detrás del predominio del tipo de lectura literal en la educación básica y media? ¿A qué se debe la ausencia generalizada de una lectura crítica intertextual? ¿Por qué la lectura se hace desde los esquemas del lector y no se presenta una negociación de sentido a partir de los esquemas propuestos por el texto? ¿Qué relación tiene lo anterior con la imposibilidad manifiesta por los estudiantes para asumir y reconocer desde la lectura diversas situaciones discursivas?

Los resultados de las pruebas aplicadas a los éstudiantes de tercero, quinto, séptimo y noveno, Pruebas Saber, indican que en la educación básica primaria y básica secundaria se privilegian prácticas de lectura literal, en detrimento de una lectura crítica intertextual, lo cual está íntimamente relacionado con ciertos modos de construir y adquirir conocimientos, procesar, comprender y comunicar información a través del lenguaje. En otras palabras, con una concepción de lenguaje a la cual subyace una concepción de conocimiento que orienta las prácticas en el aula.

A juicio del ICFES, la dificultad de los estudiantes para pasar de una lectura literal (nivel $B$ ) a una lectura analítica (nivel $C$ ) y luego a una crítica intertextual (nivel $D$ ) radica en su escasa experiencia para interactuar con los textos, buscar y generar relaciones entre los elementos locales y el sentido global de los mismos. Cuando el estudiante, en su proceso de lectura, conjetura sobre qué dice el texto, quién lo dice, cómo lo dice, para qué lo dice y cuándo lo dice, le da sentido a lo que lee y se ve en la necesidad de llenar los espacios vacíos movilizando información desde sus saberes previos y desde otros que el texto incita a conocer. Leer significa hallar y generar sentidos. Sin embargo, por el número de estudiantes que alcanza a ubicarse en los diferentes niveles de lectura de las Pruebas Saber, se puede afirmar que son muy pocos los que están acostumbrados a interrogarse mientras leen. Estas problemáticas son las que hacen que en la Prueba de Estado la mayoría de los estudiantes no logre superar los niveles medios en las competencias evaluadas. 
Entre el paso del nivel $B$ al $C$ en las Pruebas Saber está el proceso de construcción de la macro estructura de un texto. Construir la macro estructura de un texto implica la acción cooperante de un lector capaz de generar procesos de inferencia cada vez más complejos sobre la información que nos brinda el texto. Recordemos que una actividad como el resumen requiere de una dinámica de lectura e interpretación que permita identificar, seleccionar, clasificar, suprimir y generalizar información, operaciones que buscan dar cuenta sobre lo que dice el texto de manera global.

Por otra parte, trabajar sobre la gramática del texto no es reconocer o subrayar las conjunciones, preposiciones y verbos que se pueden encontrar en un escrito para ejemplificar conceptos. El trabajo sobre la gramática en los textos, desde un proceso de lectura crítico, debe responder a la función que ésta cumple en la construcción del sentido, en la organización y tejido textual. Se trata de poner lo enunciado en relación con sus enunciadores y posibles enunciatarios, para dilucidar la estructura y los mecanismos que posibilitan la coherencia y cohesión en el discurso. El trabajo sobre la organización local y global de un texto permite generar hipótesis sobre las intenciones y propósitos de los enunciadores. Todo texto responde a una organización particular, a una manera de decir que da cuenta de una semiótica de la escritura. Cuando el estudiante logra reconocer y hacer conjeturas sobre este tipo de estructuras y relaciones, amplía el sentido literal de los enunciados y provoca nuevos conocimientos sobre lo enunciado y sus enunciadores.

De esta manera, el paso entre un nivel de comprensión y otro está mediado por un tipo de operaciones y relaciones semánticas que debe dilucidar y poner en claro el lector, para lograr ubicar las unidades de sentido que el texto contiene y que permiten generar entramados o redes de sentidos posibles con otros textos. Como consecuencia de lo anterior, se hace posible la movilización de saberes de un texto en otro, ampliando el espectro de posibilidades de sentido que todo texto promueve y que el lector, en este caso el estudiante, debe estar en capacidad de resolver atendiendo a sus saberes básicos en la disciplina.

Este tipo de lectura apunta a la actividad de un sujeto discursivo que aprende el sentido del mundo a través de múltiples lenguajes; a un sujeto que reconoce en la acción del discurso formas de significar y de dar sentido al mundo. Un sujeto que lee y aprende leyendo a través de textos verbales y no verbales. Desde este enfoque, la lectura y la escritura son actos de conocimiento que no sólo permiten la interpretación de la realidad sino que, ante todo, posibilitan transformarla. Transformación que se da a través de la acción propositiva y argumentativa del lector.

Estas acciones propositivas y argumentativas se hacen inalcanzables en. el ambiente escolar cuando los lectores han sido sometidos a un proceso educativo en el que la lectura y la escritura son actividades anexas al proceso de aprendizaje. Recordemos que en la concepción tradicional de lectoescritura se hace caso omiso a esta actividad proponente del sujeto lector. Desde este enfoque, la lengua es sólo un instrumento de la comunicación, y los procesos de lectura y escritura operaciones en las que el estudiante decodifica y codifica mensajes atendiendo al buen uso de las reglas establecidas desde la gramática de la lengua como una estructura abstracta y ajena al hacer discursivo de los sujetos.

En este sentido, si escribir consiste en dominar las reglas de funcionamiento del sistema lingüístico, el trabajo pedagógico del docente se concentrará en la apropiación, por parte de los estudiantes, de estos elementos para luego ser utilizados: uno será el momento del aprendizaje y otro el de su aplicación. Las prácticas de escritura, en este caso, estarán orientadas a la mecanización de la norma y las prácticas de lectura al reconocimiento de información literal.

Por el contrario, otras son las condiciones si se concibe la escritura como un proceso complejo que implica la reelaboración permanente del significado y la búsqueda del sentido. En este caso, la norma lingüística estaría al servicio de la necesidad de la significación y la comunicación. Bajo esta misma orientación, en las clases de literatura prevalece la memorización de títulos de obras y sus autores, sin leer las obras mismas. Un saber sobre las características de los periodos y escuelas, desconociendo las obras fundamentales de dichos periodos y las condiciones discursivas que hacen posible dicha distinción en la cultura.

Es necesario resaltar que los enfoques teóricoconceptuales.inherentes a las gramáticas de cada disciplina presuponen, también, unos enfoques pedagógicos para su transposición. La metodología se construye en el proceso pero requiere de la 
apropiación de un enfoque teórico que la sustente y la oriente. En nuestro caso, por ejemplo, se habla actualmente, en los Lineamientos Curriculares y en los Indicadores de Logro, de una pedagogía que propenda por el desarrollo de una conciencia discursiva que incida en los procesos de interacción con el conocimiento a través de la lectura y la escritura. En consecuencia, resulta imposible emprender acciones de mejoramiento en el aula si los agentes involucrados desconocen desde dónde se está pensando la educación en lengua materna. Mientras el docente desconozca el enfoque que:subyace a los lineamientos curriculares, los indicadores de logro y los diferentes documentos que en los últimos años intentan dar cuenta de la evolución de las ciencias del lenguaje y su pedagogía; le será muy difícil ser proponente y crítico en el aula, $y$, por su parte, promover estos haceres en los estudiantes.

En las diferentes pruebas realizadas, el objeto de evaluación ha dado un giro de ciento ochenta grados con respecto a las que se hacían hace una década.

Desde este nuevo enfoque, todo enunciado es un acto 'social y leerlo e interpretarlo significa entender la realidad histórica y cultural que lo hace posible. En consécuencia, se busca superar la memorización mecánica de datos, fórmulas y nombres que generan una actitud pasiva en el lector, ya que su actividad se reduce a repetir lo que dice el texto por el temor de interrogarlo y sospechar sobre su contenido.

Por el contrario, los estudiantes acceden a lecturas críticas interpretativas según sean sus experiencias lectoras, pues son textos y fuentes lo que se activa cada vez que se desea leer e interpretar un texto. Cuando hablamos de textos no nos referimos sólo a aquellos que circulan en la clase de lengua y literatura, sino a todos los que ponen en contacto al estudiante con el mundo de la cultura. Es posible que la poca comprensión que se tiene sobre el enfoque que subyace a las pruebas y los lineamientos que hoy condensan la propuesta en el área de lengua castellana y literatura provengan, de una parte, de la falta de formación de los docentes en el campo de las teoríás del discurso y el texto de lingüística. Y, de otra, de la poca acción de las facultades de educación por hacer de estas teorías un marco de acción pedagógica.

Después de dos décadas de evaluaciones masivas persisten dificultades para comprender el enfoque por competencias y redimensionarlo en el aula. La cultura que pesa sobre la enseñanza de la lengua en nuestro contexto hace que a algunos docentes y padres de familia les cueste mucho trabajo comprender que lo que se evidencia en los haceres de los estudiantes son procesos del pensamiento y que, por lo tanto, la evaluación debe apuntar hacia esas estructuras complejas y no a la repetición de definiciones o conceptos que se pierden en cuanto el estudiante no les encuentra sentido para sus experiencias de vida.

Las situaciones anteriores remiten a la formación de los docentes, a los programas de las facultades de educación y a la manera como se concibe la docencia como una actividad para la aplicación de modelos. Reducir los procesos educativos a meras técnicas susceptibles de ser aplicadas en el aula, sin pasar por la mediación de la contradicción y la conjetura, inherente a la construcción de los saberes, ha constituido uno de los modelos más dominantes en la formación de los maestros. Del análisis de las prácticas de lectoescritura que realizan la mayoría de los estudiantes, se puede deducir que la excesiva importancia otorgada por al ${ }^{\llcorner}$ gunos docentes al método es una situación que se deriva de la falta de conciencia sobre la relación existente entre la práctica pedagógica y la concepción teórica y epistemológica subyacente. No se trata de acercarnos a las disciplinas desde una perspectiva meramente enciclopedista, de acumulación transitoria de información, sino de cómo ponerla en contacto con problemas que reclama su hacer en el aula en la interacción con el saber.

Es aquí donde podemos preguntar por el efecto y las necesidades de los proyectos de investigación en el campo de la pedagogía y los procesos de formación de docentes formadores de docentes.

Se trata de la competencia en el dominio disciplinario pero también de la competencia estratégica en el tratamiento de un problema concreto a partir de ese dominio de la disciplina. Por ejemplo, en el caso de las carreras en lingüística y literatura no basta con la apropiación de las teorías literarias, de poder distinguirlas y caracterizarlas, pues hasta allí podemoś hablar de un tipo de saber, necesario sin duda, pero cuyo sentido pleno se alcanza sólo en la medida en que se puedan proyectar en el análisis específico de los textos literarios; y el para qué de estos análisis cọbra importancia al vincularse con la respuesta a un problema fundamental, como lo es la lectura crítica y no literal de un texto. Una lectura que implique el 
desentrañamiento de los mundos ideológicos y culturales que hacen posible un texto que construye conocimiento y apoya el desarrollo de estructuras de pensamiento más complejas.

\section{ALGUNAS SUGERENCIAS PARA EL MEJORAMIENTO}

¿Cuáles podrían ser las posibles acciones a seguir? Siendo conscientes de la incidencia del lenguaje en los procesos de generación y construcción de conocimientos, la proyección pedagógica actual exige el desarrollo de las habilidades comunicativas en los estudiantes. Por ello, el objetivo principal de la enseñanza de la lengua materna debe apuntar a que el estudiante use el lenguaje como herramienta fundamental en la interacción con el saber. Desde esta perspectiva, una de las labores fundamentales de los docentes, sea cual sea su área de trabajo, debe ser la de propiciar el acceso a mayores niveles de dominio sobre el lenguaje. Se hace necesario, para acceder a los diferentes niveles que se proponen en la prueba, implementar el trabajo de lecturas que amplíen el saber medio de los estudiantes en la Básica. Seleccionar y trabajar con textos que le permitan al docente y al estudiante enfrentar formas de organización textual y discursiva diversas, que exigen en el lector niveles de abstracción y análisis diferentes.

Si estamos de acuerdo en que la escuela debe favorecer la adquisición y el desarrollo de las habilidades discursivas de los estudiantes, si deseamos ser coherentes con los fines comunicativos inherentes al trabajo pedagógico de quienes enseñamos lengua y literatura, habrá que empezar a entender el aula como un espacio cooperativo de creación y recepción de textos de diversa índole para entender la lengua y la literatura en el espacio de la comunicación y para la comunicación. Es importante, entonces, implementar un trabajo serio con los textos, verbales y no verbales, que circulan en el aula y por fuera de ella. El trabajar con textos que convoquen a la movilización de saberes, al pensamiento relacional y al trabajo interdisciplinar de las áreas, procura herramientas para el debate y la toma de posición frente a la construcción de conocimientos.

Reconocer que todo texto instaura una situación de comunicación y que lo que se comunica tiene una organización particular, atendiendo a unas intenciones enunciativas, genera en el estudiante una responsabilidad frente a la significación, aten- diendo al rol que ocupe en el acto comunicativo: de quien escribe o habla y de quien escucha o lee. En este sentido, trabajar un texto, ya sea para leerlo o para escribirlo, significa poner en juego y desarrollar estrategias para la construcción y deconstrucción discursiva; por ejemplo, establecer relaciones entre términos que desencadenan un tema particular, develar tópicos locales y globales, reconocer las relaciones referenciales y su secuencialidad temática en el texto, reconocer quiénes hablan y de qué estrategias discursivas se valen para organizar su discurso atendiendo a una intención comunicativa. Así, el análisis discursivo permite generar reflexiones en el estudiante sobre la manera como opera el lenguaje en el proceso de significación, y, además, le permite conocer nuevas formas de estructurar sus conocimientos y saberes.

"Atribuirle otro lugar a la lectura y escritura de textos en el aula". Este enunciado, muy socializado en diferentes espacios, presupone la necesidad de darle al texto el lugar que le corresponde en el proceso de construcción de saberes, donde la escritura y la lectura, junto con la oralidad y la escucha, son haceres fundamentales. La lectura, la discusión y la escritura deben convertirse en haceres significativos en la construcción de sentidos, es decir, en la construcción de conocimientos. El texto, debe ser el motivo, inicio y resultado de un proceso. Decir esto implica: sustraer la noción de conocimiento de su sinonimia con los contenidos. Reconocer el sentido que tiene la relación lenguaje, pensamiento y cultura en el proceso del conocimiento. Hacer del aula de clase un espacio para la significación y la recontextualización de saberes. Replantear la comunicación que se da en el aula y asignarles un nuevo rol a los participantes. En conclusión, redefinir el tan mencionado proceso enseñanza-aprendizaje y generar otras formas de evaluar y valorar el proceso del conocer y sus productos.

El trabajo de formación y de actualización con los maestros requiere apoyarse en proyectos de investigación, tanto de quienes promueven tal acción - agentes universitarios- como de quienes la demandan - los maestros-. Es desde la experiencia investigativa que se hace realidad la interlocución con los maestros. Trabajar con los maestros -y los futuros maestros- de manera interlocutiva es reconocer que hay unos saberes mínimos desde los cuales se puede despegar para cualificar progresivamente la práctica, haciéndola más lúdica y menos rutinaria. Ello implica, igual 
mente, de parte de los investigadores, participar directamente en el quehacer de la escuela, discutiendo con los maestros puntos de vista y estrategias posibles hacia la innovación: se trata, entonces, de la relación cooperativa entre la universidad y la escuela, resaltando la necesidad de que la escuela se ponga a tono con los desarrollos contemporáneos de la ciencia, la tecnología, los medios y las artes.

Finalmente, es bueno recordar que para Frank Smith la base de la comprensión es la predicción o la eliminación previa de alternativas improbables... las predicciones son preguntas que formulamos al mundo, la comprensión es recibir respuestas. Si no podemos predecir, estamos confundidos. Y si no tenemos nada que predecir, porque no tenemos incertidumbre, estaremos aburridos.

\section{BUBLIOGRAFÍA}

BAENA, Luis Ángel, (1989), "El lenguaje y la significación”, "Funciones del lenguaje y enseñanza de la lengua", "Estructura, funcionamiento y función", Revista Lenguaje, Cali, No. 17, Universidad del Valle, diciembre.

-, (1992), "Actos de significación", Revista Lenguaje, Cali, No. 19 y 20 , Universidad del Valle.

BAJTíN, M. M., (1999), Estética de la creación verbal, México, $10{ }^{a}$ edición, Siglo XXI Editores.

BARTHES, Roland, (1987), "De la ciencia a la liteŕratura", en: El susurro del lenguaje, Barcelona, Paidós.

-, (1973), El grado cero de la escritura, México, Siglo XXI.

BENVENISTE, Emile, (1987), "Semiología de la lengua", en: Problemas de lingüística general II, México, Siglo XXI.

BERGER, P. y Luckmann T. , (1968), La construcción social de la realidad, Buenos Aires, Amorrortu.

BERNSTEIN, Basil, (1990), La construcción social del discurso pedagógico, Bogotá, El Griot.

-, (2000), Hacia una sociología del discurso pedagógico, Bogotá, Magisterio.

BRUNER, Jerome, (1988), Realidad mental y mundos posibles, Barcelona, Gedisa.

DIJK van, Teun A., (1977), "La pragmática de la comunicación literaria", en: J. A. Mayoral (ed.), s. l.

-, (1978), La ciencia del texto. Un enfoque interdisciplinario, Barcelona, Paidós.

-, (1980), Estructuras y funciones del discurso, México, Siglo XXI.

DURKHEIM, Emil, (1988), "El papel de las universidades en la educáción social del país”, en: Durkheim, Educación y pedagogía, Bogotá, ICFES-UPN.

ECO, Umberto, (1965), “El problema de la recepción”, en: AA. VV., Sociología contra psicoanálisis, Barcelona, Martínez Roca.
-, (1972), La estructura ausente, Barcelona, Lumen.

-, (1977), Tratado de semiótica general, Barcelona, Lumen.

-, (1981), Lector in fabula, Barcelona, Lumen.

-, (1985), Obra abierta, Barcelona, Ariel.

-, (1988), Signo, Barcelona, Labor.

-, (1995), Los límites de la interpretación, Barcelona, Lumen.

GENETTE, Gérard, (1989), Palimpsestos. La literatura en segundo grado, Madrid, Taurus.

GREIMAS, A. J., (1971), Semántica estructural. Investigación metodológica, Madrid, Gredos.

_, (1983), La semiótica del texto: ejercicios prácticos, Barcelona, Paidós.

JURADO, Fabio, (1998), Investigación, escritura y educación, Bogotá, Universidad Nacional.

—y Bustamante Guillermo, (1997), Los procesos de la escritura, Bogotá, Magisterio.

MAGLI, Patrizia, Manetti Giovanni, y Violi Patricia (eds.), (1992), Semiotica: storia, teoria, interpretazione. Saggi intorno a Umberto Eco, Milán, Bompiani.

MORALES V., Rosa y Blanca Bojacá, (2002), ¿Qué hacemos los maestros cuando hablamos en el aula?, Bogotá, Universidad Distrital Francisco José de Caldas, Colciencias.

ONG, Walter, (1987), Oralidad y escritura, México, Fondo de Cultura Económica.

PEIRCE, Charles Sanders, (1987), Obra Lógico-semiótica, Madrid, Taurus.

-, (1988) El hombre, un signo. El pragmatismo de Peirce, Barcelona, Crítica.

SILVESTRI, Adriana y Blanck Guillermo, (1993), Bajtín y Vigotski: la organización semiótica de la conciencia, Barcelona, Anthropos.

SISTEMA NACIONAL DE EVALUACIÓN DE LA EDUCACIÓN (SNE), (1997), Evaluación de logros, Áreas de Lenguaje y Matemáticas, 1992-1994, Bogotá, Serie Publicaciones para Maestros, Men.

SMITH, Frank, (1983), Comprensión de la lectura, México, Trillas.

-, (1999), Evaluación de logros, Áreas de Lenguaje y Matemáticas 1992, 1994 y 1997, Bogotá, Serie Nuevo Examen de Estado, Área de Lenguaje.

TODOROv, Tzvetan, (1983), Gramática del Decamerón, Madrid, Josefina Betancor.

-, (1991), Crítica de la crítica, Barcelona, Paidós.

_, (1993), Las morales de la historia, Barcelona, Paidós. 